\title{
Controversies in therapeutic application of mesenchymal stem cell-derived secretome
}

\author{
FERENC SIPOS*; GYÖRGYI MÜZES \\ Department of Internal Medicine and Hematology, Semmelweis University, Budapest, 1088, Hungary
}

Key words: Mesenchymal stem cells, Secretome, Exosomes, Cancer

\begin{abstract}
Though mesenchymal stem cells (MSCs) are considered as an important pillar of regenerative medicine, their regenerative potential has been shown to be limited in several pathological conditions. The adverse properties of MSCbased cell therapy have drawn attention to the therapeutic use of MSC-derived secretome. However, MSC-originated exosomes and microvesicles can also possess a significant impact on disease development, including cancer. By interchanging secretome, MSCs can interact with tumor cells and promote mutual exchange/induction of cellular markers. In addition, enzymes secreted into and activated within exosomes can result in the acquisition of new tumor cell properties. Therefore, therapeutic applications of MSC-derived secretome require much caution.
\end{abstract}

\section{Introduction}

Stem cell research and tissue engineering seem to be integral parts in regenerative medicine. According to their ability to differentiate into new cell lines, stem cells can be classified as totipotent, pluripotent, multipotent, and unipotent ones. The use of allogeneic cells may lead to complications, including immunological rejection, but upon administration of autologous cells rejection can be avoided, thus it represents a relatively safer therapeutic form.

Of the adult stem cells, hematopoietic stem cells and mesenchymal stem/stromal cells (MSCs) are the most frequently used ones, mainly because they can be obtained from patients suffering from distinct disease conditions (e.g., aplastic anemia, Duchenne muscular dystrophy, ankylosing spondylitis, etc.) (Vasanthan et al., 2020).

MSCs possess the potential for self-renewal along with limited capacity of differentiation. Their main sources are bone marrow, adipose tissue, skin, liver, lungs, cord blood, and fallopian tube (Mohammadian et al., 2013).

The therapeutical usage of MSCs faces issues like the difficulty of maintaining a homogenous culture and, further, characterizing the cells (Meirelles et al., 2009). Besides cell replacement function, MSCs have a wide range of biological effects (i.e., immunomodulation, anti-apoptotic and antifibrotic activity, angiogenesis, chemoattraction, pro-growth

${ }^{\star}$ Address correspondence to: Ferenc Sipos, sipos.ferenc@med.semmelweis-univ.hu

Received: 06 July 2021; Accepted: 30 August 2021 and pro-differentiation activity on other stem cells) (Chang et al., 2006; Jones et al., 2007; Saeedi et al., 2019; Patel et al., 2013). One of their most promising properties is the secretion of bioactive components, known as secretome, into the cell culture conditioned medium (González-González et al., 2020). The secretome consists of a soluble and a vesicular fraction. The soluble fraction is rich in cytokines, chemokines, growth factors, and immunomodulatory molecules. The vesicular fraction contains extracellular vesicles, which according to size and synthesis route could be subdivided to exosomes, microvesicles and apoptotic bodies. The MSC-derived secretome consists of exosomes and microvesicles, which in turn contain proteins, lipids, or nucleic acids (González-González et al., 2020). The secretome can directly activate the target cells partly by endocytosis and may exert a broad spectrum of actions, as indicated above (Hassanpour et al., 2020). Nevertheless, it is important to note, that the therapeutic capacity of the secretome may vary depending on the origin of MSCs (Zhao et al., 2019).

\section{Difficulties in Mesenchymal Stem Cell-Based Therapies}

Based on their broad spectrum of functional properties, MSCs can be considered as a relevant pillar for regenerative medicine, therefore, their biosafety characteristics during clinical use should be critical in order to eliminate potential functional or genetic changes. According to various studies, however, the regenerative potential of MSCs has been shown to be very limited, especially within pathological 
conditions. Although MSCs are present in several tissues, their number is quite small. In addition, the viability of transplanted cells and their uptake into host tissues are often diminished (Haque et al., 2015). Moreover, many factors, like the age of the donor, the number of passages and culture conditions in the course of in vitro expansion, the route of administration, and the pathological host microenvironment confronted by the transplanted MSCs may unfavorably impact on the capacity of these cells to survive and engraft into host tissues (Rezaie et al., 2018). Recent studies have also indicated possible pro-tumorigenic activities of MSCs (Barkholt et al., 2013) along with pro-fibrogenic and pro-coagulant potentials (Russo et al., 2006; Fischer et al., 2009), an increased risk of infections (e.g., zoonotic diseases) during the in vitro expansion procedure (Lepperdinger et al., 2008), and the unfavorable heterogeneity of their differentiation potential (Fig. 1) (McLeod and Mauck, 2017).

\section{Mesenchymal Stem Cells' Secretome: Disadvantages in the Shadow of Advantages}

The adverse properties of MSC-based cell therapy have drawn attention to the therapeutic use of the MSCs' secretome. Application of the MSC-derived secretome has many meaningful advantages, including the lack of need for surgical intervention to recover cells, the possibility of drug-dose dosage and safety evaluations, the easier way of administration, or the potential manipulation on its composition (Baglio et al., 2012). MSC-derived soluble and vesicular factors represent numerous special properties that could make them an invaluable tool for therapeutic applications (González-González et al., 2020). However, concrete issues arise when working with these entities, whose physical and biochemical characteristics often make them difficult to obtain as pure preparations, and to characterize correctly. Therefore, the International Society for Extracellular Vesicles (ISEV) proposed Minimal Information for Studies of Extracellular Vesicles ("MISEV") guidelines for the field in 2014, which has been recently revised in 2018 (Théry et al., 2018).

On the other hand, exosomes can also possess a significant impact on disease development, including cancer. Tissue damage results in the recruitment of MSCs to support wound healing and regeneration. Similarly, invasive tumor growth also leads to (in part inflammatory) tissue damage with the consequence of intensive attraction and cellular cross-modulation. By interchanging secretome, MSCs can interact with tumor cells and promote mutual exchange/induction of cellular markers (Yang et al., 2015; Nawaz et al., 2018; Mandel et al., 2013; Hass and Otte, 2012; Yang et al., 2015; Salimi et al., 2020).

In addition to the direct effect of soluble fraction secreted by MSCs, enzymes secreted into and activated within exosomes (mainly matrix metalloproteinases /MMP/ and MMP regulators) can result in the acquisition of new tumor cell properties (Yang et al., 2015). The vesicular fraction of secretome plays a role in the construction of pre-metastatic niche and tumor neovascularization. Furthermore, extracellular-matrix-associated abnormalities could affect cancer progression by promoting fibroblastic switching and acquisition of mesenchymal mode (Nawaz et al., 2018).

The incorporation of MSC-derived exosomes has been shown to be associated with acquired ecto-5'-nucleotidase activity by certain tumor cells (Yang et al., 2015). With this new capability tumor cells can suppress and modify proinflammatory activities (e.g., tumor-infiltrating T-cell function) via activation of adenosine receptor signaling present on the surface of most immune cells (Ohta and Sitkovsky, 2014; Clayton et al., 2011).

Vice versa, tumor cells can also affect and modify MSCs through their secretome (Nawaz et al., 2018; Ma et al., 2020). Extracellular vesicles secreted by cancerous (stem) cells are able to develop a pre-metastatic niche and induce epidermalmesenchymal transition, favoring tumor spreading (Fig. 2) (Nawaz et al., 2018).

In addition to unwanted biological properties, vesicular secretome isolation techniques (i.e., ultracentrifugation, membrane filtration, precipitation, size exclusion chromatography, immunoaffinity capture technology) are also currently cumbersome, generally resulting in small amounts of lowpurity, sometimes deformed extracellular vesicles. Their subsequent use is therefore difficult to implement (Ma et al., 2020; Ahmadi et al., 2021; Ahmadi and Rezaie, 2021; Babaei and Rezaie, 2021).

\section{Future Perspectives}

According to ClinicalTrials.gov the number of studies using MSC-derived secretome is quite low (i.e., 10), and only 3
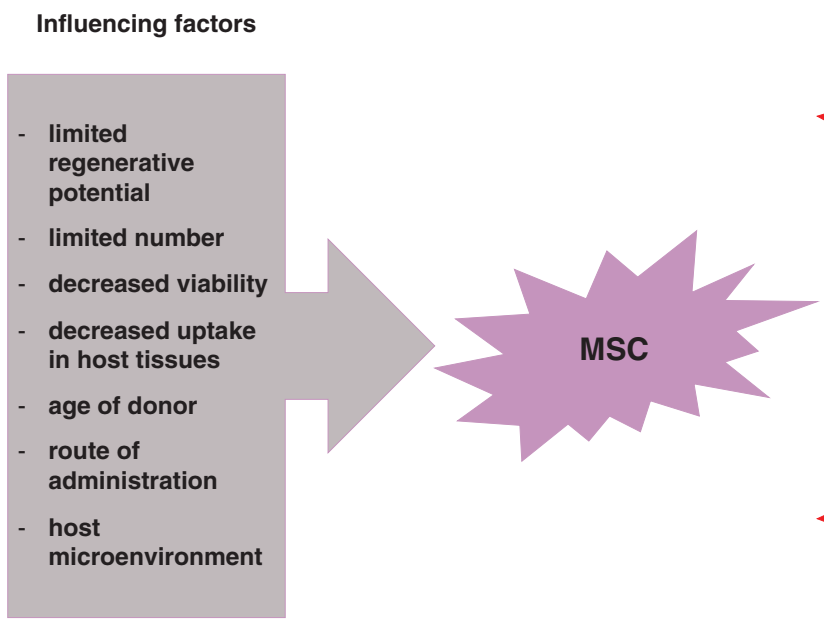

FIGURE 1. Factors influencing the therapeutic potential of mesenchymal stem cells and the resulting adverse effects. 

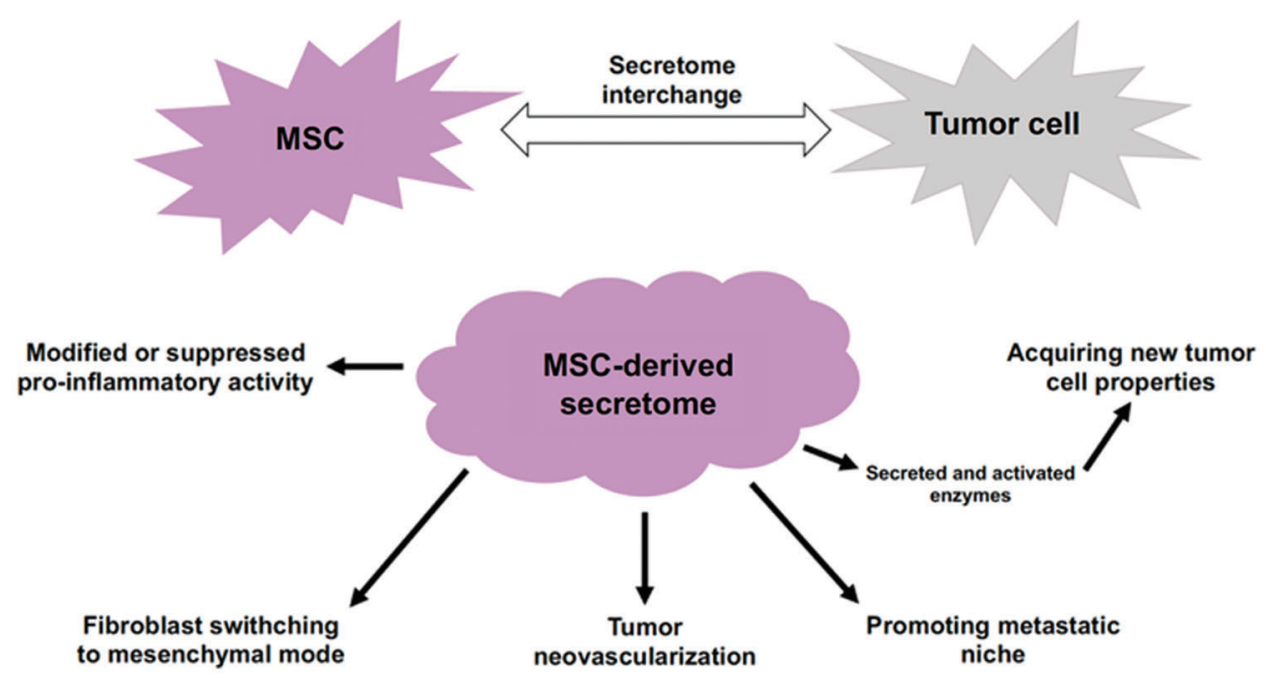

FIGURE 2. Unfavorable effects of secretome interchange between mesenchymal stem cell and tumor cells.

have been completed up-to-date. The therapeutic application of MSC-derived secretome looks promising, yet caution is required. It is not just about that the composition and function of the MSC-derived secretome are highly dependent on the origin of the MSCs (i.e., healthy, inflammatory or tumorous environment), but for the time being, the therapeutic targeting of the secretome used is also difficult (Phelps et al., 2018). Whichever route of administration is used, it is not yet possible to be absolutely certain that the bioactive substance will act on a particular cell type, nor is it entirely possible to determine how the expected biological effect is affected by the milieu to which the secretome is added.

Currently we also lack knowledge of how the MSCs and their secretome can be affected by the combinations of drugs used in the disease conditions. Further researches are also needed to explore how possible genetic or epigenetic changes in MSCs affect the composition and biological effects of the secretome. This is especially important to avoid possible tumorigenic side-effects (Hassanzadeh et al., 2021).

In addition to the technical difficulties of finding and extracting MSCs, new and efficient laboratory techniques are also needed to extract the MSC-derived secretome in the right quantity and quality, making it easier to apply in daily practice. It would be useful to reduce the time and cost of these new techniques as well, thus efficiently promoting their spread. Overall, there is no doubt that besides the cellular-based strategies cell-free bioactive materials, like the secretome may represent a considerable alternative in translational medicine.

Acknowledgement: We thank Anika Scott for her assistance.

Availability of Data and Materials: No data are included within this viewpoint.

Author Contribution: The author confirms sole responsibility for the following: study conception and design, data collection, analysis and interpretation of results, and manuscript preparation.

Funding Statement: This work was supported by the StartUp Program of Semmelweis University Faculty of Medicine (CO Nos. 11720, Ikt.sz.: 5127/AOKGIE/2018; SE10332470).
Conflicts of Interest: The authors declare that they have no conflicts of interest to report regarding the present study.

\section{References}

Ahmadi M, Jafari R, Mahmoodi M, Rezaie J (2021). The tumorigenic and therapeutic functions of exosomes in colorectal cancer: Opportunity and challenges. Cell Biochemistry and Function 39: 468-477. DOI 10.1002/cbf.3622.

Ahmadi M, Rezaie J (2021). Ageing and mesenchymal stem cells derived exosomes: Molecular insight and challenges. Cell Biochemistry and Function 39: 60-66. DOI 10.1002/ cbf.3602.

Babaei M, Rezaie J (2021). Application of stem cell-derived exosomes in ischemic diseases: Opportunity and limitations. Journal of Translational Medicine 19: H1439. DOI 10.1186/s12967-02102863-w.

Baglio SR, Pegtel DM, Baldini N (2012). Mesenchymal stem cell secreted vesicles provide novel opportunities in (stem) cellfree therapy. Frontiers in Physiology 3: 359. DOI 10.3389/ fphys.2012.00359.

Barkholt L, Flory E, Jekerle V, Lucas-Samuel S, Ahnert P et al. (2013). Risk of tumorigenicity in mesenchymal stromal cell-based therapies-bridging scientific observations and regulatory viewpoints. Cytotherapy 15: 753-759. DOI $10.1016 / \mathrm{j}$. jcyt.2013.03.005.

Chang CJ, Yen ML, Chen YC, Chien CC, Huang HI et al. (2006). Placenta-derived multipotent cells exhibit immunosuppressive properties that are enhanced in the presence of interferongamma. Stem Cells (Dayton, Ohio) 24: 2466-2477. DOI 10.1634/stemcells.2006-0071.

Clayton A, Al-Taei S, Webber J, Mason MD, Tabi Z (2011). Cancer exosomes express CD39 and CD73, which suppress T cells through adenosine production. Journal of Immunology 187: 676-683. DOI 10.4049/jimmunol.1003884.

Fischer UM, Harting MT, Jimenez F, Monzon-Posadas WO, Xue H et al. (2009). Pulmonary passage is a major obstacle for intravenous stem cell delivery: The pulmonary first-pass effect. Stem Cells and Development 18: 683-692. DOI 10.1089/scd.2008.0253.

González-González A, García-Sánchez D, Dotta M, Rodríguez-Rey JC, Pérez-Campo FM (2020). Mesenchymal stem cells secretome: The cornerstone of cell-free regenerative 
medicine. World Journal of Stem Cells 12: 1529-1552. DOI 10.4252/wjsc.v12.i12.1529.

Haque N, Kasim NH, Rahman MT (2015). Optimization of pretransplantation conditions to enhance the efficacy of mesenchymal stem cells. International Journal of Biological Sciences 11: 324-334. DOI 10.7150/ijbs.10567.

Hass R, Otte A (2012). Mesenchymal stem cells as all-round supporters in a normal and neoplastic microenvironment. Cell Communication and Signaling 10: 26. DOI 10.1186/ 1478-811X-10-26.

Hassanpour M, Rezabakhsh A, Rezaie J, Nouri M, Rahbarghazi R (2020). Exosomal cargos modulate autophagy in recipient cells via different signaling pathways. Cell \& Bioscience 10: 37. DOI 10.1186/s13578-020-00455-7.

Hassanzadeh A, Rahman HS, Markov A, Endjun JJ, Zekiy AO et al. (2021). Mesenchymal stem/stromal cell-derived exosomes in regenerative medicine and cancer; overview of development, challenges, and opportunities. Stem Cell Research \& Therapy 12: 331. DOI 10.1186/s13287-021-02378-7.

Jones BJ, Brooke G, Atkinson K, McTaggart SJ (2007). Immunosuppression by placental indoleamine 2,3-dioxygenase: A role for mesenchymal stem cells. Placenta 28: 1174-1181. DOI 10.1016/j.placenta.2007.07.001.

Lepperdinger G, Brunauer R, Jamnig A, Laschober G, Kassem M (2008). Controversial issue: Is it safe to employ mesenchymal stem cells in cell-based therapies? Experimental Gerontology 43: 10181023. DOI 10.1016/j.exger.2008.07.004.

Ma ZJ, Yang JJ, Lu YB, Liu ZY, Wang XX (2020). Mesenchymal stem cell-derived exosomes: Toward cell-free therapeutic strategies in regenerative medicine. World Journal of Stem Cells 12: 814-840. DOI 10.4252/wjsc.v12.i8.814.

Mandel K, Yang Y, Schambach A, Glage S, Otte A et al. (2013). Mesenchymal stem cells directly interact with breast cancer cells and promote tumor cell growth in vitro and in vivo. Stem Cells and Development 22: 3114-3127. DOI 10.1089/scd.2013.0249.

McLeod CM, Mauck RL (2017). On the origin and impact of mesenchymal stem cell heterogeneity: New insights and emerging tools for single cell analysis. European Cells and Materials 34: 217-231. DOI 10.22203/eCM.v034a14.

Meirelles L, Fontes AM, Covas DT, Caplan AI (2009). Mechanisms involved in the therapeutic properties of mesenchymal stem cells. Cytokine \& Growth Factor Reviews 20: 419-427. DOI 10.1016/j.cytogfr.2009.10.002.

Mohammadian M, Shamsasenjan K, Lotfi Nezhad P, Talebi M, Jahedi $\mathrm{M}$ et al. (2013). Mesenchymal stem cells: New aspect in cellbased regenerative therapy. Advanced Pharmaceutical Bulletin 3: 433-437. DOI 10.5681/apb.2013.070.

Nawaz M, Shah N, Zanetti BR, Maugeri M, Silvestre RN et al. (2018). Extracellular vesicles and matrix remodeling enzymes: The emerging roles in extracellular matrix remodeling, progression of diseases and tissue repair. Cells 7: 167. DOI $10.3390 /$ cells7100167.

Ohta A, Sitkovsky M (2014). Extracellular adenosine-mediated modulation of regulatory $\mathrm{T}$ cells. Frontiers in Immunology 5: 267. DOI 10.3389/fimmu.2014.00304.

Patel DM, Shah J, Srivastava AS (2013). Therapeutic potential of mesenchymal stem cells in regenerative medicine. Stem Cells International 2013: 1-15. DOI 10.1155/2013/496218.

Phelps J, Sanati-Nezhad A, Ungrin M, Duncan NA, Sen A (2018). Bioprocessing of mesenchymal stem cells and their derivatives: Toward cell-free therapeutics. Stem Cells International 2018: 1-23. DOI 10.1155/2018/9415367.

Rezaie J, Mehranjani MS, Rahbarghazi R, Shariatzadeh MA (2018). Angiogenic and restorative abilities of human mesenchymal stem cells were reduced following treatment with serum from diabetes mellitus type 2 patients. Journal of Cellular Biochemistry 119: 524-535. DOI 10.1002/jcb.26211.

Russo FP, Alison MR, Bigger BW, Amofah E, Florou A et al. (2006). The bone marrow functionally contributes to liver fibrosis. Gastroenterology 130: 1807-1821. DOI 10.1053/j.gastro.2006.01.036.

Saeedi P, Halabian R, Imani Fooladi AA (2019). A revealing review of mesenchymal stem cells therapy, clinical perspectives and Modification strategies. Stem Cell Investigation 6: 34. DOI 10.21037/sci.2019.08.11.

Salimi L, Akbari A, Jabbari N, Mojarad B, Vahhabi A et al. (2020). Synergies in exosomes and autophagy pathways for cellular homeostasis and metastasis of tumor cells. Cell \& Bioscience 10: 37. DOI 10.1186/s13578-020-00426-y.

Théry C, Witwer KW, Aikawa E, Alcaraz MJ, Anderson JD et al. (2018). Minimal information for studies of extracellular vesicles 2018 (MISEV2018): A position statement of the International Society for Extracellular Vesicles and update of the MISEV2014 guidelines. Journal of Extracellular Vesicles 7: 1535750. DOI 10.1080/20013078.2018.1535750.

Vasanthan J, Gurusamy N, Rajasingh S, Sigamani V, Kirankumar S et al. (2021). Role of human mesenchymal stem cells in regenerative therapy. Cells 10: 54. DOI 10.3390/cells10010054.

Yang Y, Bucan V, Baehre H, von der Ohe J, Otte A et al. (2015). Acquisition of new tumor cell properties by MSC-derived exosomes. International Journal of Oncology 47: 244-252. DOI 10.3892/ijo.2015.3001.

Yang Y, Otte A, Hass R (2015). Human mesenchymal stroma/stem cells exchange membrane proteins and alter functionality during interaction with different tumor cell lines. Stem Cells and Development 24: 1205-1222. DOI 10.1089/scd.2014.0413.

Zhao T, Sun F, Liu J, Ding T, She J et al. (2019). Emerging role of mesenchymal stem cell-derived exosomes in regenerative medicine. Current Stem Cell Research \& Therapy 14: 482494. DOI 10.2174/1574888X14666190228103230. 